available methods, some of which may be useless for the analysis of complex substances.

For each element or group of elements, information is given concerning behaviour during the course of an 'ordinary' analysis, methods of decomposing minerals, methods of separation and methods of determination. The book has been brought up to date by appropriate omissions, additions and rewriting; special methods, such as those involving the use of the spectrograph, the polarograph, etc., receive attention, but are not described in detail. Some information is given about nearly all the chemical elements, the only elements not included being inappropriate ones, that is, the rare gases, actinium, polonium and the elements discovered during recent years. The section on the rare-earth metals contains much useful information concerning the more orthodox methods, but ion-exchange methods are not described in detail ; however, this section, like all sections of the book, is adequately annotated with references to other publications. Similar comments apply to other parts of the book; as examples, the dithizone method for lead, and ether-extraction methods for the separation of uranium are not described in detail. In this edition, the sections dealing with glass, bouxite and refractories have been omitted, but the classical methods used by Dr. Hillebrand for rock and mineral analysis are retained, with necessary additions.

C. O. Harvey

\section{A MANUAL OF SCIENTIFIC AND INDUSTRIAL INSTRUMENTS}

The Instrument Manual

Second edition. Pp. vii $+628+105$. (London: United Trade Press, Ltd., 1953.) 848.

\section{1} THE writing of an adverse review is always an unpleasant task, but the unpleasantness increases with the size and price of the book concerned; there seems to be an instinctive feeling that a large and expensive volume should be more reliable than a small, cheap one.

The second edition of the "Instrument Manual" is designed on a large and expensive scale; the idea behind it is excellent, but the result is sadly dis. appointing. The manual is divided into a series of chapters each of which deals with a particular type of measurement and consists of a theoretical introduction, descriptions of the instruments available, a list of manufacturers and $a_{0}$ list of references to the literature. The field covered is very wide and the present reviewer can only comment with confidence on the detail of one or two of the chapters, but grossly misleading and erroneous statements found on familiar subjects undermine one's confidence in the remainder.

Perhaps examples from one of the more unfortunate sections might be quoted here (p. 56, Polarimetry) : "Polarisation. To understand polarisation it is necessary to regard light as being a wave motion in more than one plane, e.g. there will be transverse vibrations as well as those in the direction of travel of the wave. The effect is indicated simply in Fig. OP 11. If it should be possible to remove one of these modes of vibration, the wave is said to have become plane polarised". The figure mentioned is said to represent a wave travelling along the $z$-axis of a system of co-ordinates and shows two sinusoidal waves, one along $z$ and one along $y$, at right angles to it. Later in the paragraph we find the statement : "If $y$ becomes zero, the wave can be said to be plane polarised . . .". Towards the end of the following section we read: "If the plane polarised light emerging from a Nicol prism is allowed to fall on a second prism with its principal plane parallel to the first, the beam is split into two components, one of which, as in the first Nicol, is transmitted. If the second prism is rotated, it will be found that the intensity of the light decreases to a minimum when the principal planes are crossed at an angle of $90^{\circ}$. The intensity is thus proportional to the angle of rotation" (the italics are the reviewer's). These statements surely need no comment.

In addition to serious errors of this type, the book contains a large number of paragraphs which give no useful information at all and which might very well mislead a person with no previous knowledge of the instrument described. An example of this is the description of wet- and dry-bulb thermometers on p. 531. It is quoted in full : "These are mercury" in-glass thermometers in which the bulb is circular, and consist of the normal thermometer stem encased in an outer glass sheath, terminating at its upper end in a glass button for suspension purposes. The sheath not only protects the graduation against damage but prevents the condensation of moisture on the divisions or figures of the scale. At the lower end it is fused to the thermometer proper between the bulb and the lowest graduation". Such a description would certainly merit no marks at all in an examination at even the lowest level.

Some of the sections seem to be incomplete and lack continuity, as though they had been made up by stringing together the work of different writers and draughtsmen without proper co-ordination. For example, in a section of the chapter on temperature measurement headed "Black Body Radiation" (which, incidentally, begins "If a piece of carbon or charcoal (i.e. a black body) ...") we find a diagram (p. 277) of a square enclosure labelled $A$ with a ray, starting from wall $C$ and being regularly reflected in turn from walls $D, E$ and $B$. The whole paragraph relating to this figure runs as follows: "Accepting a black body condition as standard, it is desirable to achieve this as nearly as possible in actual instrumentation. Let us consider in Fig. T.T. 64 an enclosure A at a uniform temperature with a very small opening at B. The walls of the enclosure are of a material which partially absorbs and reflects radiation. A ray starting from wall $\mathrm{C}$ strikes wall $\mathrm{D}$. Corrections may be necessary because of temperature gradient in the tube".

It would not serve any useful purpose to give any more detailed examples. One might summarize by stating that the book is reminiscent of an examination script sent in by a reasonably good student who, in his anxiety to impress the examiner, has written down all he knows regardless of its relevance to the question, putting in padding where his knowledge fails, occasionally going off the rails altogether, and certainly leaving no time for reading through at the end. In the stress of an examination this could be forgiven, but one scarcely expects it in the second, revised edition of a book costing four guineas.

There is undoubtedly a need for a book covering this ground and a great opportunity has been missed. Let us hope that if a third edition should be contemplated, the attention of the editors will be concentrated on putting right what is already there rather than on the addition of new material.

C. A. TAYLOR 